\title{
El cerebro tríadico y su relación con la curiosidad, el trabajo en equipo y la explicación de fenómenos para el desarrollo de actitud científica
}

\author{
Carolina Pérez-Carrero*, Sandra Milena Rodríguez-Moreno ${ }^{* *}$, Lida del Pilar Sánchez-Mayorga ${ }^{* * *}$
}

Magíster en Educación. Profesora, Colegio Cundinamarca IEd, Bogotá, Colombia. Correo electrónico:

carito9606@yahoo.es.

Magíster en Educación. Profesora, Colegio Cundinamarca IED, Bogotá, Colombia. Correo electrónico:

sandy191277@yahoo.com

*** Magíster en Educación. Profesora, Colegio Cundinamarca IED, Bogotá, Colombia. Correo electrónico: lidadelpisama@yahoo.es

Recibido: 21 de noviembre del 2014 Aprobado: 19 de junio del 2015

Cómo citar este artículo: Pérez-Carrero, Carolina, Sandra Milena Rodríguez-Moreno y Lida del Pilar Sánchez-Mayorga. "El cerebro tríadico y su relación con la curiosidad, el trabajo en equipo y la explicación de fenómenos para el desarrollo de actitud científica". Rastros Rostros 17.31 (2015): 99-110. Impreso. doi: http://dx. doi. org/10.16925/ra.v17i31.1106

\section{Resumen}

Propósito: este artículo presenta la relación entre tres actitudes científicas: la curiosidad, el trabajo en equipo y la explicación de fenómenos con el cerebro tríadico propuesto por Waldemar De Gregory en Construcción familiar-escolar de los tres cerebros. Descripción: se propone y realiza una caracterización de dichas actitudes científicas en 30 niños de grado segundo del Colegio Cundinamarca IED en las clases de ciencias, a fin de aportar a la implementación de estrategias que fortalezcan la actitud científica de los estudiantes y contribuir, de este modo, al aprendizaje de las ciencias naturales. Punto de vista: dicha investigación, con enfoque cualitativo, descriptivo e interpretativo, pone de manifiesto la importancia de renovar las estrategias metodológicas tradicionales, en tanto que el fortalecimiento de las actitudes desde el aula permite el desarrollo de procesos de pensamiento científico. Conclusiones: de esta manera, se generarán espacios de interpretación y transformación de la realidad desde las primeras edades de los niños y las niñas.

Palabras clave: actitud científica, cibernética social, curiosidad, evolución tricerebral, explicación de fenómenos, trabajo en equipo. 


\title{
The triadic brain and its relationship with curiosity, teamwork, and explanation of phenomena in the development of a scientific attitude
}

\begin{abstract}
Purpose: this article analyzes the relationship between three scientific attitudes (curiosity, teamwork and explanation of phenomena) and the triadic brain proposed by Waldemar de Gregori in Construcción familiar-escolar de los tres cerebros [Family-school construction of the three brains]. Description: a characterization of scientific attitudes in 30 second graders in the science classroom at the Colegio Cundinamarca IED is proposed and performed to contribute to the implementation of strategies that strengthen the scientific attitude of students and thus contribute to learning Natural Sciences. Point of view: such research, with a qualitative, descriptive and interpretive approach, highlights the importance of renewing traditional methodological strategies since the strengthening of attitudes from the classroom enables the development of scientific thought processes. Conclusions: therefore, spaces for interpretation and transformation of reality from the earliest ages of children will be created.
\end{abstract}

Keywords: scientific attitude, social cybernetics, curiosity, triadic brain evolution, explanation of phenomena, teamwork.

\section{O cérebro trino e sua relação com a curiosidade, o trabalho em equipe e a explicação de fenômenos para o desenvolvimento de atitude científica}

\section{Resumo}

Propósito: este artigo apresenta a relação entre três atitudes científicas: a curiosidade, o trabalho em equipe e a explicação de fenómenos com o cérebro trino proposto por Waldemar De Gregory em Construção familiar-escolar dos três cérebros. Descrição: propõe-se e realiza uma caraterização de tais atitudes científicas em 30 crianças de segunda série do Colégio Cundinamarca IED nas aulas de Ciências, para contribuir à aplicação de estratégias que fortaleçam a atitude científica dos estudantes e contribuir, dessa forma, ao aprendizado das Ciências Naturais. Ponto de vista: essa investigação, com enfoque qualitativo, descritivo e interpretativo, evidencia a importância de renovar as estratégias metodológicas tradicionais, enquanto que o fortalecimento das atitudes desde a sala de aulas permite o desenvolvimento de processos de pensamento científico. Conclusões: dessa forma, serão gerados os espaços de interpretação e transformação da realidade desde as primeiras idades das crianças.

Palavras-chave: atitude científica, cibernética social, curiosidade, evolução tri-cerebral, explicação de fenómenos, trabalho em equipe. 


\section{Introducción}

La educación científica, entendida como la formación de individuos con autonomía y capacitados para investigar los fenómenos de la naturaleza y de su entorno, requiere de parte de la escuela primaria atención a la promoción de actitudes científicas en sus educandos, así como su contribución en la formación del espíritu científico necesario en el medio colombiano. Por lo tanto, es importante tener una visión integral del nivel de estas actitudes en los niños, con el fin de establecer posteriormente estrategias que favorezcan su fortalecimiento. Este artículo presenta los resultados de la fase de investigación del trabajo de grado "Fortalecimiento de actitudes científicas como la curiosidad, el trabajo en equipo y la explicación de fenómenos en la enseñanza de las ciencias naturales en básica primaria: el caso de los niños de segundo grado del Colegio Cundinamarca IED, Ciudad Bolívar, jornada mañana", en el cual se propone y realiza una caracterización de la curiosidad, el trabajo en equipo y la explicación de fenómenos, como actitudes científicas en relación con el cerebro tríadico que facilitan una visión integral del ser humano.

En esta investigación las actitudes científicas se definen, de acuerdo con Harlen, como "predisposiciones hacia las actividades implicadas en las ciencias, como el uso de las pruebas, la creación de ideas y el tratamiento de los ambientes naturales y artificiales de determinadas formas" (88). En este sentido, el fortalecimiento delas actitudes científicas permite el acercamiento al medio natural a través de la exploración y la indagación.

Las actitudes que se caracterizan son la curiosidad, la disposición para el trabajo en equipo y la explicación de fenómenos. En primer lugar, según Harlen, la curiosidad se hace evidente desde la elaboración de preguntas acerca del entorno inmediato; esto en la medida en que satisface sus dudas e inquietudes. Es la primera actitud científica que un niño posee y ha dado lugar a los grandes descubrimientos científicos.

Giordán indica cómo la estimulación de la curiosidad en el aprendizaje se traduce en motivación. A través de este sentimiento, los estudiantes se inquietan por buscar respuesta a sus interrogantes (en últimas son los que dan sentido a su proceso de formación en la escuela). Igualmente, la curiosidad es motor para la construcción de los procesos de pensamiento e incide en el alcance del aprendizaje, es decir, de acuerdo con el nivel de interés y curiosidad, es posible o no continuar en la búsqueda de respuestas a lo largo de los años de escolaridad, lo cual trasciende al ámbito científico.

En segundo lugar, según la fundamentación conceptual del área de ciencias naturales, realizada por el Instituto Colombiano para el Fomento de la Educación Superior (ICFES), el trabajo en equipo es una capacidad que permite interactuar productivamente al asumir compromisos. El resultado de un trabajo en equipo se centra en la construcción conjunta de un producto o de un discurso sobre un tema de estudio: "Para lograr esta construcción es preciso saber argumentar las posiciones personales y valorar y aceptar los argumentos de otros cuando se reconoce en ellos pertinencia y validez" (22). Si se tiene en cuenta lo anterior, es posible afirmar que el trabajo en equipo está ligado al aprendizaje cooperativo, en el cual se trabaja en conjunto a fin de lograr metas comunes y los integrantes se esmeran por obtener buenos resultados, tanto para sí mismos, como para los demás miembros del grupo.

Para Johnson, "el aprendizaje cooperativo es el empleo didáctico de grupos reducidos en los que los alumnos trabajan juntos para maximizar su propio aprendizaje y el de los demás" (1). Sucede lo contrario en el aprendizaje de tipo competitivo o individualista, en el cual los estudiantes compiten por obtener la mejor nota y trabajan de manera individual, con el propósito de conseguir resultados en el aprendizaje desvinculados de los demás. Es posible que esto genere en los docentes algunas limitaciones en el momento de proponer espacios en los que los estudiantes puedan utilizar sus saberes de manera apropiada, mientras que el aprendizaje cooperativo se emplea en cualquier tarea, área o programa de estudios.

Para el ICFES, el ejercicio del trabajo colectivo le ofrece al estudiante la posibilidad de aprender a expresarse libremente en una discusión, así como de desarrollar la capacidad de reconocer contextos y características propias de los participantes que le permitan llegar a proponer soluciones consensuadas. Por tanto, es necesario que se abran espacios a fin de mostrar diversas formas de ver y de afrontar una situación, reconocer que cada uno de los integrantes del grupo tiene cosas que decir y aportar al trabajo, lo cual le permite, en palabras de Velandia, "reconocer al otro como un auténtico otro" (5).

Además, el trabajo en equipo en el aula es una oportunidad para que el estudiante adquiera una serie de hábitos sociales de gran importancia para la vida cotidiana: el respeto a las opiniones de los demás, la aceptación y el cumplimiento de responsabilidades 
específicas, el buen uso del lenguaje, la selección de momentos adecuados para participar en una reunión y el sentido de pertenencia e identidad con los valores. Dichos valores permiten el enriquecimiento personal y grupal, en pro del desarrollo de comunicaciones asertivas en la escuela que luego trascienden a la familia y la sociedad.

La última actitud que se tendrá en cuenta es la explicación de fenómenos de la naturaleza, la cual, de acuerdo con Baquero, es "la capacidad para construir y comprender argumentos, representaciones o modelos que den razón de fenómenos" (18). Lo anterior se refiere a la oportunidad que tiene el ser humano de expresar los pensamientos, conocimientos y apreciaciones de los fenómenos que suceden en su contexto, los cuales generalmente son plasmados a través de explicaciones sencillas pero argumentadas, las cuales finalmente establecen una cadena de razones acerca de las causas y consecuencias de dicho fenómeno. A fin de comunicar estas explicaciones, es necesario hacer uso de los conocimientos previos y asociarlos con los conocimientos adquiridos.

Los niños elaboran las explicaciones con el propósito de dar respuesta a los fenómenos de su entorno, en concordancia con su edad, el ambiente inmediato en el cual se encuentran inmersos, las experiencias vividas, su bagaje cultural, los saberes previos y la exposición que tienen a la información ofrecida por los medios de comunicación y las Tic.

Según el ICFES:

[...] la competencia explicativa fomenta en el estudiante una actitud analítica que le posibilite establecer la validez o coherencia de una afirmación o de un argumento. Para evaluar esta competencia en la prueba se han incluido preguntas en las cuales el estudiante debe seleccionar la explicación más adecuada para dar razón de un problema o de una situación particular, deducir la validez de un argumento a partir de los referentes conceptuales que posee, o que se presentan en el enunciado, $o$ a partir de la búsqueda de relaciones y conexiones entre fenómenos y conceptos. (21)

Con frecuencia, los estudiantes argumentan sus ideas de manera sencilla utilizando un vocabulario concreto adquirido en su vida cotidiana, a partir de las experiencias vividas, la interrelación con otros y los conceptos elaborados con base en aprendizajes significativos. De la misma manera, pueden expresar su acuerdo o desacuerdo frente a las ideas de sus compañeros de manera argumentada, y así enriquecer sus propios aprendizajes.

En diversas ocasiones, el trabajo de los científicos se ha ligado al hemisferio izquierdo del cerebro, en el cual se encuentran las funciones lógicas. Olvidan que somos seres integrales y cómo en cada cosa que realizamos en nuestra vida, además de manifestarse el componente lógico, también están presentes la afectividad, la creatividad y el instinto, cualidades del cerebro triuno propuesto por De Gregori, fundamento de la propuesta de la caracterización que a continuación se presenta.

Las actitudes científicas se caracterizan de acuerdo con la perspectiva de la evolución tricerebral, fundamentada en la cibernética social. De acuerdo con De Gregori, esta es la ciencia, el arte y la técnica de la autoorganización, de la autoinformación y de la autoconducción o gobernabilidad de cualquier persona o grupo en los tres campos estratégicos del juego de la vida, es decir, en el pensar, en el sentir y en el actuar. En este sentido, este planteamiento consiste en la gestión de toda la manifestación tríadica del ser humano.

La cibernética social se pone en práctica a partir de la dinámica de grupos en los que se usan liderazgos. En estos, cada uno de los integrantes del grupo tiene unas funciones específicas: por ejemplo, llevar el tiempo, hacer una motivación o evaluar la actividad. Una característica primordial consiste en usar referenciales tales como cuadros de doble entrada, esquemas y mapas mentales, a fin de representar de manera gráfica el discurso teórico y, de esta manera, organizar la información. Uno de los referenciales más utilizados es el ciclo cibernético de transformación, definido por De Gregori como un ciclo en el que se ensamblan tres operaciones: saber, hacer y crear. Es cibernético porque da cuenta del sistema focalizado y de la transformación que el ciclo cibernético busca regular, al cambiar la dirección de la marcha evolutiva-transformativa que acontece en el mundo.

Con la cibernética social, se obtienen diversas herramientas que permiten desarrollar el gran y diverso potencial que habita en los cerebros de los seres humanos. Para ello se debe comprender que el cerebro límbico - de acuerdo con la denominación de McLean-, corresponde al cerebro derecho, emocional o intuitivo (Velandia), el cual responde al ser en su esencia, sus valores y su espiritualidad. El neocórtex corresponde al cerebro izquierdo y lógico; responde a los procesos lógicos, al análisis, la crítica y la reflexión. Y por último, el cerebro reptiliano 
corresponde al cerebro central, el cual responde a los procesos operativos o prácticos.

Las habilidades, operaciones y facultades mentales son muchas. Por el cociente tríadico, es decir, por el potencial mental, son 28 . Pero es importante empezar por ensamblar las tres operaciones mínimas (saber, hacer y crear), operación que se denomina ciclo cibernético de transformación (ССт) mínimo. Se menciona que forman un "ciclo" porque este gira sin fin, después de uno y otro, a manera de espiral. "Cibernético", porque informa del estado del sistema focalizado, y de "transformación", porque el ciclo cibernético busca regular y direccionar la marcha evolutiva-transformativa en que se mueve el mundo (De Gregori). Es así como se propone en este artículo relacionar tres actitudes científicas con las operaciones mínimas o el cerebro triuno. En primer lugar, la curiosidad con la creación o el cerebro creativo; luego, el trabajo en equipo con el hacer o el cerebro operativo; y por último, la explicación de fenómenos con el saber o el cerebro lógico. De esta manera, se busca - a través de la caracterización de las actitudes científicas de 30 niños de grado segundo del Colegio Cundinamarca IED-, una visión más amplia y menos restringida en comparación con la que se obtiene cuando la investigación se enfoca solo en las funciones lógicas del cerebro. Esto permite además diseñar diversas estrategias en concordancia con el saber, el hacer y el crear, y así favorecer el aprendizaje de las ciencias y motivar el espíritu científico.

En los siguientes apartados se explica cómo se realiza la caracterización de acuerdo con la evolución tricerebral, y se presentan los hallazgos encontrados, el análisis y las conclusiones.

\section{Metodología}

La presente investigación se basa en la metodología cualitativa con enfoque descriptivo e interpretativo, la cual, de acuerdo con Hernández, Fernández y Baptista, permite dar profundidad a los datos, al interpretar y generar una contextualización de detalles y experiencias que aportan al punto de vista holístico de dichos fenómenos.

Dicha metodología se basa en la descripción de variables a evaluar, en este caso las actitudes científicas (curiosidad, explicación de fenómenos y disposición para el trabajo en equipo). Se diseñaron tres instrumentos, a saber: registro de observación directa; el revelador del cociente tríadico y las concepciones de los docentes de ciclo uno frente a la estimulación de las actitudes científicas.
En el registro de observación se describen los niveles de evolución de los 30 niños de grado segundo frente a cada una de las actitudes científicas, en cada una de las clases de ciencias naturales. Teniendo en cuenta que cada actitud se relaciona directamente con cada uno de los cerebros (teoría tricerebral de De Gregori), se establecen los criterios de observación. Así, en la curiosidad se determina la interacción del niño con los objetos del entorno; en cuanto al trabajo en equipo, se analiza la interacción social; y, finalmente, en la explicación de fenómenos, se tienen en cuenta las expresiones verbales. Es decir, cada actitud es observada en los cuatro niveles de evolución tricerebral. A continuación se presenta la tabla 1 , la cual corresponde a la matriz de registro de observación aplicada a cada uno de los 30 niños, en las tres sesiones de las clases de ciencias naturales. Cada sesión se diseñó a partir de actividades específicas orientadas a observar cada una de las actitudes en los cuatro niveles de evolución tricerebral, a través de la unidad temática "Animales y su hábitat". Cada sesión corresponde a un momento de la actividad, lo cual permitió describir en detalle las características de los niños en virtud de la información requerida en dicho instrumento de observación.

Para la lectura, la interpretación y el análisis de las observaciones, se diseñó la matriz en términos de competencias, con el fin de medir los niveles de la evolución tricerebral por niño y por grupo, lo cual, de acuerdo con De Gregori, permite desarrollar armónicamente los tres lados del cerebro. A continuación se presentan los resultados en la tabla 2 .

En segundo lugar, el revelador del cociente triádico permite determinar la dominancia cerebral, la cual se traduce en las funciones lógicas, operativas y creativas, a fin de establecer un equilibrio en el aprendizaje a través de la estimulación de la función menos desarrollada, y así mejorar significativamente la actuación del ser humano en la sociedad (De Gregori). A partir de la presente investigación es posible plantear estrategias metodológicas, las cuales permitan fortalecer las funciones cerebrales menos desarrolladas de los niños de grado segundo y alcanzar de este modo una educación más integral.

De Gregori menciona cómo, para que la educación sea "más efectiva, tendrá que disminuir el enfoque hacia el currículo y darle centralidad al desarrollo del potencial tricerebral" (84); es decir, la educación desde las ciencias naturales debe iniciarse en la adquisición y fortalecimiento de procesos, en este caso orientados hacia las actitudes científicas y no un aprendizaje centrado en contenidos aislados. 
En tercer lugar, se aplica una encuesta a los catorce docentes de Ciclo 1 de la institución, con el fin de determinar en la fase diagnóstica sus concepciones relativas a las actitudes científicas y a las estrategias que las fortalecen desde la escuela. Este instrumento consta de seis preguntas abiertas, las cuales permiten analizar la utilización de recursos para la clase de ciencias naturales, la importancia de cada una de las actitudes científicas y cómo se evidencian en los estudiantes de Ciclo 1 en general.

Tabla 1. Registro de observación

\begin{tabular}{|c|c|c|c|c|c|c|c|c|c|c|c|c|}
\hline \multirow{2}{*}{$\frac{\text { Muestra: }}{\text { Objetivo: }}$} & \multicolumn{4}{|c|}{ Fecha: } & \multicolumn{2}{|l|}{ Hora: } & \multicolumn{5}{|c|}{ Lugar: } & \\
\hline & & & & & & & & & & & & \\
\hline \multicolumn{13}{|c|}{ Descripción actitudes científicas } \\
\hline \multirow{3}{*}{ Momentos } & \multicolumn{4}{|l|}{ Curiosidad } & \multicolumn{4}{|c|}{ Trabajo en equipo } & \multicolumn{4}{|c|}{ Explicación de fenómenos } \\
\hline & \multicolumn{4}{|c|}{ Interacción con los objetos del entorno } & \multicolumn{4}{|c|}{ Interacción social } & \multicolumn{4}{|c|}{ Expresiones verbales } \\
\hline & $\begin{array}{l}\text { Observa- } \\
\text { ción de } \\
\text { elemen- } \\
\text { tos del } \\
\text { entorno } \\
\text { inmediato }\end{array}$ & $\begin{array}{l}\text { Manipula- } \\
\text { ción } \\
\text { De objetos } \\
\text { regis- } \\
\text { trando } \\
\text { informa- } \\
\text { ciones }\end{array}$ & $\begin{array}{l}\text { Experimen- } \\
\text { tación con } \\
\text { elementos } \\
\text { del entorno } \\
\text { haciendo } \\
\text { predicciones }\end{array}$ & $\begin{array}{l}\text { Diseño y } \\
\text { construc- } \\
\text { ción de } \\
\text { modelos } \\
\text { explicativos }\end{array}$ & $\begin{array}{l}\text { Segui- } \\
\text { miento de } \\
\text { instruccio- } \\
\text { nes }\end{array}$ & $\begin{array}{l}\text { Escucha } \\
\text { activamente } \\
\text { a los demás }\end{array}$ & $\begin{array}{l}\text { Interpreta } \\
\text { las opinio- } \\
\text { nes de los } \\
\text { demás y } \\
\text { establece } \\
\text { acuerdos }\end{array}$ & $\begin{array}{l}\text { Desarrolla } \\
\text { actividades } \\
\text { en equipo } \\
\text { a través } \\
\text { de la sana } \\
\text { conviven- } \\
\text { cia }\end{array}$ & $\begin{array}{l}\text { Expone } \\
\text { ideas, pen- } \\
\text { samientos, } \\
\text { sensacio- } \\
\text { nes }\end{array}$ & $\begin{array}{l}\text { Expresa } \\
\text { puntos } \\
\text { de vista } \\
\text { acerca del } \\
\text { contexto }\end{array}$ & $\begin{array}{l}\text { Argumenta } \\
\text { ideas y } \\
\text { opiniones }\end{array}$ & $\begin{array}{l}\text { Analiza } \\
\text { fenómenos } \\
\text { eviden- } \\
\text { ciando } \\
\text { su propio } \\
\text { criterio }\end{array}$ \\
\hline \multicolumn{13}{|l|}{$\begin{array}{l}\text { Primer } \\
\text { Momento }\end{array}$} \\
\hline \multicolumn{13}{|l|}{$\begin{array}{l}\text { Segundo } \\
\text { Momento }\end{array}$} \\
\hline $\begin{array}{l}\text { Tercer } \\
\text { Momento }\end{array}$ & & & & & & & & & & & & \\
\hline
\end{tabular}

Fuente: elaboración propia.

Tabla 2. Niveles de evolución tricerebral

\begin{tabular}{|c|c|c|c|}
\hline & $\begin{array}{l}\text { Pensar-saber } \\
\text { Cerebro izquierdo } \\
\text { Explicación de fenómenos }\end{array}$ & $\begin{array}{l}\text { Hacer-tener } \\
\text { Cerebro central } \\
\text { Trabajo en equipo }\end{array}$ & $\begin{array}{l}\text { Sentir-ser } \\
\text { Cerebro derecho } \\
\text { Curiosidad }\end{array}$ \\
\hline Mentalización & $\begin{array}{l}\text { Analizar fenómenos del entorno, } \\
\text { usando el lenguaje gráfico, escrito } \\
\text { y verbal, evidenciando criterios } \\
\text { propios y aplicándolos en otros } \\
\text { contextos y situaciones. }\end{array}$ & $\begin{array}{l}\text { Desarrollar actividades en equipo, } \\
\text { adoptando un comportamiento } \\
\text { responsable, constructivo y solidario, } \\
\text { que fortalezca una sana convivencia } \\
\text { con sus pares y el entorno, como } \\
\text { criterio clave para hacer ciencia. }\end{array}$ & $\begin{array}{l}\text { Construir maquetas o modelos } \\
\text { sencillos que expliquen fenómenos } \\
\text { o situaciones cotidianas aplicando } \\
\text { los diferentes conocimientos } \\
\text { interiorizados en el aula. }\end{array}$ \\
\hline Asesoría & $\begin{array}{l}\text { Argumentar ideas y opiniones } \\
\text { frente a situaciones que se proponen } \\
\text { en el contexto. }\end{array}$ & $\begin{array}{l}\text { Interpretar las opiniones de los } \\
\text { demás, reconociendo puntos de vista } \\
\text { diferentes. }\end{array}$ & $\begin{array}{l}\text { Experimentar con elementos y } \\
\text { situaciones del entorno haciendo } \\
\text { predicciones, tomando en cuenta } \\
\text { variables planteadas. }\end{array}$ \\
\hline Supervisión & $\begin{array}{l}\text { Expresar puntos de vista acerca de } \\
\text { situaciones del contexto, con base } \\
\text { en conocimientos previos. }\end{array}$ & $\begin{array}{l}\text { Escuchar activamente a los pares para } \\
\text { la identificación de ideas, teniendo en } \\
\text { cuenta el respeto y la tolerancia. }\end{array}$ & $\begin{array}{l}\text { Manipular objetos del entorno } \\
\text { registrando información, de } \\
\text { acuerdo con una tabla de datos. }\end{array}$ \\
\hline Ejecución & $\begin{array}{l}\text { Exponer de manera espontánea } \\
\text { ideas, pensamientos y emociones } \\
\text { para relacionarse con sus compañe- } \\
\text { ros, teniendo en cuenta la experien- } \\
\text { cia de la vida diaria. }\end{array}$ & $\begin{array}{l}\text { Seguir instrucciones para la ela- } \\
\text { boración de trabajos individuales } \\
\text { y grupales, de acuerdo con lo que } \\
\text { indica el tutor. }\end{array}$ & $\begin{array}{l}\text { Observar elementos y situacio- } \\
\text { nes del entorno inmediato para } \\
\text { reconocerlo, tomando en cuenta } \\
\text { indicaciones recibidas. }\end{array}$ \\
\hline
\end{tabular}

Fuente: elaboración propia a partir de los niveles de evolución tricerebral de De Gregori, Waldemar. Construcción familiar-escolar de los tres cerebros. Bogotá: Editorial Kimpres Ltda., 2002. Impreso.

En segundo lugar, el revelador del cociente triádico permite determinar la dominancia cerebral, la cual se traduce en las funciones lógicas, operativas y creativas, a fin de establecer un equilibrio en el aprendizaje a través de la estimulación de la función menos desarrollada, y así mejorar significativamente la actuación del ser humano en la sociedad (De Gregori). A partir de la presente investigación es posible plantear estrategias metodológicas, las cuales permitan fortalecer las funciones cerebrales menos 
desarrolladas de los niños de grado segundo y alcanzar de este modo una educación más integral.

De Gregori menciona cómo, para que la educación sea "más efectiva, tendrá que disminuir el enfoque hacia el currículo y darle centralidad al desarrollo del potencial tricerebral" (84); es decir, la educación desde las ciencias naturales debe iniciarse en la adquisición y fortalecimiento de procesos, en este caso orientados hacia las actitudes científicas y no un aprendizaje centrado en contenidos aislados.

En tercer lugar, se aplica una encuesta a los catorce docentes de Ciclo 1 de la institución, con el fin de determinar en la fase diagnóstica sus concepciones relativas a las actitudes científicas y a las estrategias que las fortalecen desde la escuela. Este instrumento consta de seis preguntas abiertas, las cuales permiten analizar la utilización de recursos para la clase de ciencias naturales, la importancia de cada una de las actitudes científicas y cómo se evidencian en los estudiantes de Ciclo 1 en general.

Tabla 3. Niveles de evolución tricerebral para la curiosidad
Finalmente se realiza la triangulación de resultados, contrastando la evidencia de cada uno de los instrumentos aplicados a los estudiantes, con el fin de describir e interpretar el nivel de cada actitud científica, así como las perspectivas de los docentes en relación con estas.

\section{Resultados}

\section{Registro de observación}

Por medio del uso del registro de observación, se recolectó la información que relaciona las tres actitudes científicas (curiosidad, trabajo en equipo e interpretación de fenómenos). Esta última se analiza a partir de los cuatro niveles de evolución tricerebral (ejecución, supervisión, asesoría y mentalización), tal como se muestra en las tablas 2, 3 y 4, así como en las figuras $1,2,3$ y 4 .

\begin{tabular}{|c|c|c|c|}
\hline \multicolumn{4}{|c|}{ CURIOSIDAD } \\
\hline \multicolumn{4}{|c|}{ Interacción con los objetos del entorno } \\
\hline Nivel de ejecución. & Nivel de supervisión. & Nivel de asesoría. & Nivel de mentalización. \\
\hline $\begin{array}{l}\text { Observación de elementos del entorno } \\
\text { inmediato. }\end{array}$ & $\begin{array}{c}\text { Manipulación } \\
\text { de objetos registrando } \\
\text { informaciones. }\end{array}$ & $\begin{array}{l}\text { Experimentación con } \\
\text { elementos del entorno } \\
\text { haciendo predicciones. }\end{array}$ & $\begin{array}{l}\text { Construcción de modelos } \\
\text { explicativos. }\end{array}$ \\
\hline $\begin{array}{l}\text { No tienen contacto visual ni táctil con } \\
\text { los animales. } \\
\text { Muestran interés por la actividad del } \\
\text { animal. } \\
\text { De acuerdo con la observación que } \\
\text { hicieron generaron preguntas. } \\
\text { Se mostraron apáticos por observar los } \\
\text { animales. } \\
\text { Se observaron sentimientos de emoción } \\
\text { en algunos niños, y en otros de rechazo. }\end{array}$ & $\begin{array}{l}\text { Interactúan con los animales } \\
\text { sacándolos de su hábitat, tocán- } \\
\text { dolos con gusto y jugando con } \\
\text { ellos. Se negaron a tocar a los } \\
\text { animales. } \\
\text { Registran en una tabla de datos } \\
\text { el comportamiento del animal. } \\
\text { La información registrada no } \\
\text { corresponde a lo solicitado en } \\
\text { la tabla. }\end{array}$ & $\begin{array}{l}\text { Con base en la documen- } \\
\text { tación predicen en que } \\
\text { estadio se encuentran los } \\
\text { animales. } \\
\text { Predicen qué les puede } \\
\text { suceder a los animales si se } \\
\text { les cambia las condiciones } \\
\text { de vida. } \\
\text { Predicen la forma de repro- } \\
\text { ducción. }\end{array}$ & $\begin{array}{l}\text { A través de dibujos plas- } \\
\text { man las condiciones de } \\
\text { vida de su animal. } \\
\text { Hacen dibujos relativos al } \\
\text { animal pero no expresan } \\
\text { ninguna explicación con } \\
\text { respecto a un proceso. } \\
\text { No construyen modelos } \\
\text { que expliquen algún } \\
\text { proceso. }\end{array}$ \\
\hline
\end{tabular}

Fuente: elaboración propia a partir de los niveles de evolución tricerebral de De Gregori, Waldemar. Construcción familiar-escolar de los tres cerebros. Bogotá: Editorial Kimpres Ltda., 2002. Impreso.

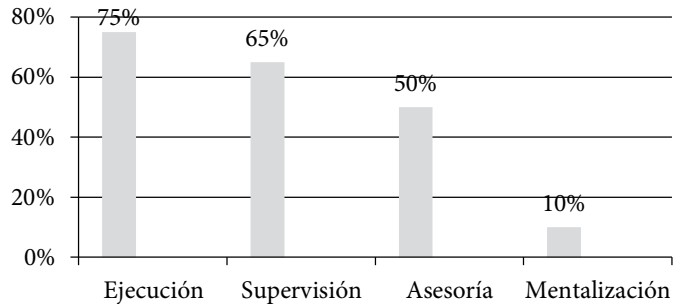

Figura 1. Porcentaje de estudiantes en los niveles de evolución tricerebral con respecto a la curiosidad.
En la figura 1 se muestra que la mayoría de los estudiantes (75\%) se muestran motivados e interactúan espontáneamente con su entorno. Algunos (65\%) interactúan con sus animales y registran la información requerida en las tablas de datos de manera adecuada. Pocos estudiantes (50\%) realizan predicciones teniendo en cuenta los conceptos trabajados con anterioridad. Muy pocos estudiantes (10\%) evidencian sus conocimientos y proporcionan explicaciones de sus experiencias a través de dibujos. 
Tabla 4. Niveles de evolución tricerebral en la disposición para trabajar en equipo

\begin{tabular}{|c|c|c|c|}
\hline \multicolumn{4}{|c|}{ Trabajo en Equipo } \\
\hline \multicolumn{4}{|c|}{ Interacción social } \\
\hline Nivel de ejecución. & Nivel de supervisión. & Nivel de asesoría. & Nivel de mentalización. \\
\hline $\begin{array}{l}\text { Seguimiento de instruc- } \\
\text { ciones. }\end{array}$ & $\begin{array}{l}\text { Escucha activamente a los } \\
\text { demás. }\end{array}$ & $\begin{array}{l}\text { Interpreta las opiniones de los } \\
\text { demás. }\end{array}$ & $\begin{array}{l}\text { Desarrolla actividades en equipo a } \\
\text { través de la sana convivencia. }\end{array}$ \\
\hline $\begin{array}{l}\text { No siguen las indicaciones } \\
\text { en el orden apropiado. } \\
\text { No interpretan instruccio- } \\
\text { nes verbales ni escritas. } \\
\text { De un total de instruccio- } \\
\text { nes solo siguen algunas. }\end{array}$ & $\begin{array}{l}\text { No escuchan a sus compañeros. } \\
\text { No aceptan la opinión de los } \\
\text { demás. } \\
\text { Interrumpen a sus compañeros } \\
\text { cuando hablan. } \\
\text { Hablan al tiempo o cuando otro } \\
\text { está hablando. }\end{array}$ & $\begin{array}{l}\text { No comprenden las ideas que } \\
\text { sus compañeros expresan. } \\
\text { Repiten ideas ya dichas por } \\
\text { sus compañeros. } \\
\text { No son capaces de decir con } \\
\text { sus palabras lo que otro ha } \\
\text { dicho. }\end{array}$ & $\begin{array}{l}\text { Se evidencian claramente los tres } \\
\text { roles del juego tríadico. } \\
\text { No logran llegar a acuerdos. } \\
\text { Con dificultad realizan un pro- } \\
\text { ducto grupal. }\end{array}$ \\
\hline
\end{tabular}

Fuente: elaboración propia a partir de los niveles de evolución tricerebral de De Gregori, Waldemar. Construcción familiar-escolar de los tres cerebros. Bogotá: Editorial Kimpres Ltda., 2002. Impreso.

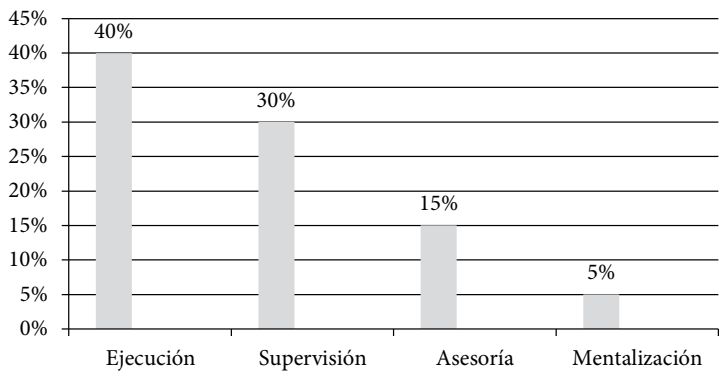

Figura 2. Porcentaje de los niveles de evolución tricerebral de los estudiantes con respecto a la disposición para trabajar en equipo.

Fuente: elaboración propia.

De acuerdo con la figura 2, al 40\% de los estudiantes generalmente se les dificulta seguir instrucciones para elaborar trabajos, tanto individuales como grupales. El 30\% de los estudiantes no no tienen el hábito de la escucha y por consiguiente les cuesta identificar las ideas de los demás. En general, el 15\% de los estudiantes no dan valor a las opiniones de sus compañeros, ya que ponen en primer lugar las propias. Se ve una dificultad ya que solo el $5 \%$ de los estudiantes es capaz de compartir, respetar ideas y asignarse roles.

De acuerdo con la figura 3, se observa en los estudiantes como el 66,6\% expresa de manera espontánea lo que siente y pregunta sobre lo que le interesa. El 93,3\% manifiesta interés por expresar sus opiniones, pero generalmente sin establecer relaciones de la temática con el entorno. El $23,3 \%$ da ideas y opiniones pero sin justificar ni argumentar. El 6,6\% de los estudiantes no evidencia criterios propios para analizar hechos, conceptos y fenómenos.

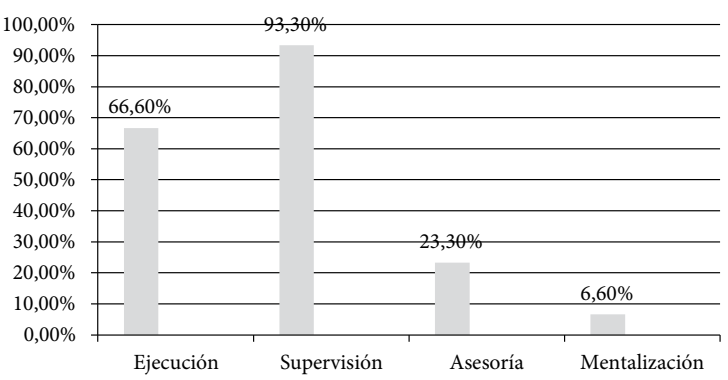

Figura 3. Porcentaje de los niveles de evolución tricerebral de los estudiantes con respecto a la explicación de fenómenos.

Fuente: elaboración propia.

\section{Revelador del cociente mental tríadico}

La aplicación del revelador del cociente mental tríadico permite establecer la dominancia cerebral en la población de estudio, como se evidencia en la figura 4 .

De acuerdo con la figura 4, el predominio cerebral de los estudiantes se concentra en el lado derecho con un $63,30 \%$, de los cuales el $33 \%$ son niñas y el $30 \%$ niños. En segundo lugar de predominancia se encuentra el cerebro izquierdo con un $20 \%$ de estudiantes, del cual el $13 \%$ son niños y el $6,60 \%$ niñas. En último lugar se encuentra el cerebro central con un $16,60 \%$ de estudiantes, del cual todos son niños. 
Tabla 5. Niveles de evolución tricerebral para la explicación de fenómenos

\begin{tabular}{|c|c|c|c|}
\hline \multicolumn{4}{|c|}{ Explicación de fenómenos } \\
\hline \multicolumn{4}{|c|}{ Expresiones verbales } \\
\hline Nivel de ejecución & Nivel de supervisión & Nivel de asesoría & Nivel de mentalización \\
\hline $\begin{array}{l}\text { Expone ideas, pensamientos, } \\
\text { sensaciones. }\end{array}$ & $\begin{array}{l}\text { Expresa puntos de vista acerca } \\
\text { del contexto. }\end{array}$ & Argumenta ideas y opiniones. & $\begin{array}{l}\text { Analiza hechos y fenóme- } \\
\text { nos evidenciando su propio } \\
\text { criterio. }\end{array}$ \\
\hline $\begin{array}{l}\text { Expresan de manera espontá- } \\
\text { nea lo que sienten. } \\
\text { Hacen preguntas sobre cosas } \\
\text { les interesan. } \\
\text { Guardan silencio. }\end{array}$ & $\begin{array}{l}\text { Expresan ideas que no tienen } \\
\text { coherencia con lo que se está } \\
\text { hablando. } \\
\text { Expresan ideas sencillas sobre } \\
\text { el tema. } \\
\text { No establecen conexiones de } \\
\text { la temática trabajada con el } \\
\text { entorno. }\end{array}$ & $\begin{array}{l}\text { Justifican las respuestas que } \\
\text { dan. } \\
\text { Las ideas y opiniones no son } \\
\text { justificadas. }\end{array}$ & $\begin{array}{l}\text { No presentan criterios propios } \\
\text { para analizar un fenómeno. }\end{array}$ \\
\hline
\end{tabular}

Fuente: elaboración propia a partir de los niveles de evolución tricerebral de De Gregori, Waldemar. Construcción familiar-escolar de los tres cerebros. Bogotá: Editorial Kimpres Ltda., 2002. Impreso.
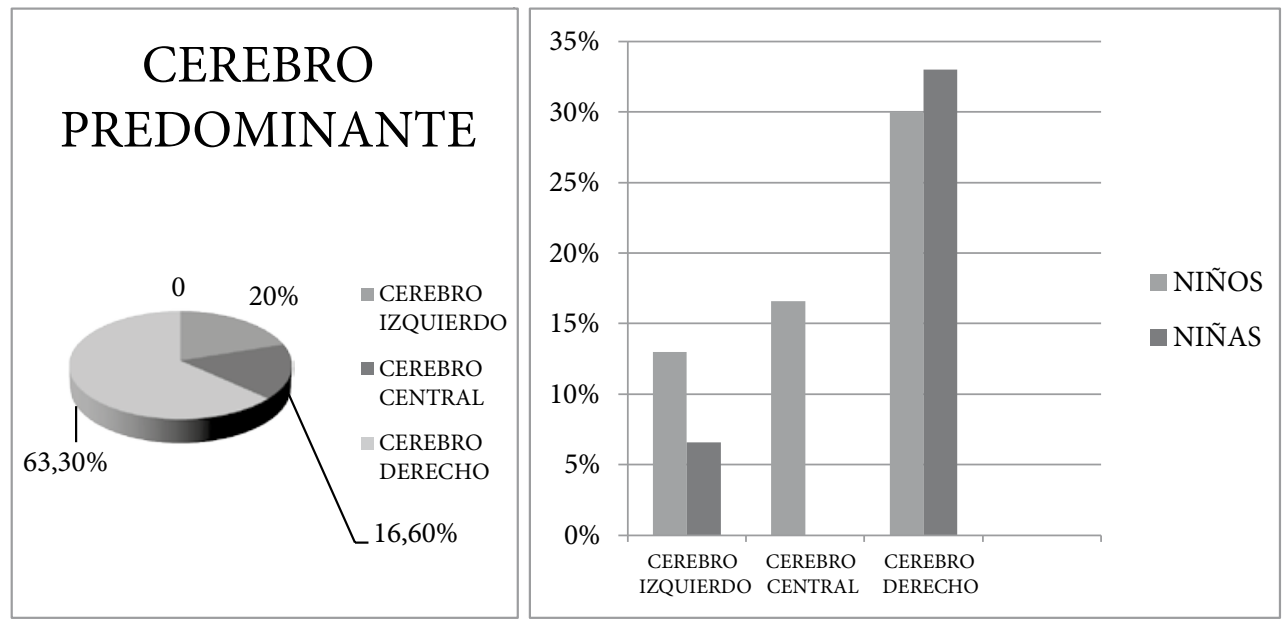

Figura 4. Predominio cerebral de los estudiantes.

Fuenete: elaboración propia.

De acuerdo con la figura 4, el predominio cerebral de los estudiantes se concentra en el lado derecho con un $63,30 \%$, de los cuales el $33 \%$ son niñas y el 30\% niños. En segundo lugar de predominancia se encuentra el cerebro izquierdo con un $20 \%$ de estudiantes, del cual el $13 \%$ son niños y el $6,60 \%$ niñas. En último lugar se encuentra el cerebro central con un $16,60 \%$ de estudiantes, del cual todos son niños.

\section{Encuesta a docentes}

A través de la aplicación de una encuesta a los 14 docentes de Ciclo 1 (docentes de grados preescolar, primero y segundo), del Colegio Cundinamarca, se recolectó información sobre sus percepciones acerca de la definición de actitudes científicas en general, definición de curiosidad, trabajo en equipo y explicación de fenómenos; las estrategias que usan para incentivar las actitudes científicas y cómo las evidencian en sus estudiantes. Dicha encuesta se estructuró en seis preguntas abiertas, de tal manera que se hizo evidente la percepción de cada uno de los docentes.

Entre los resultados se encontró que los docentes saben qué es curiosidad. Este elemento lo corroboran a través de las preguntas que los niños generan y el interés por explorar y manipular los elementos del 
entorno. Además, la mayoría de los docentes manifiesta que utiliza asiduamente el trabajo en equipo como estrategia; sin embargo, es evidente que no hay claridad en este concepto, aunque algunos consideran que no es posible trabajar en equipo en razón a la edad de los niños. Los docentes frecuentemente organizan a los niños en grupos pero no realizan ningún trabajo en equipo. Generalmente realizan las clases en el salón, pese a que consideran que las zonas verdes, la ludoteca y la biblioteca son espacios que favorecen el desarrollo adecuado de las clases. En relación con la explicación de fenómenos dicen contrastarla por medio de expresiones o argumentos frente a situaciones específicas. No obstante, la mayoría no tiene claro el concepto de explicación de fenómenos.

\section{Análisis de resultados}

Después de analizar los resultados, se muestra un bajo nivel de las tres actitudes científicas que se pretenden fortalecer. A continuación se presenta la caracterización de estas en los cuatro niveles de evolución tricerebral.

En el nivel de ejecución, se observa el porcentaje más alto en relación con la curiosidad y el trabajo en equipo, dado que los niños en estas edades se encuentran en la etapa de las operaciones concretas, la cual se caracteriza, según Daza, por la forma particular de aprender que desarrollan; esto es, por medio de la relación directa con su entorno obtienen sus conocimientos como resultado de experiencias significativas (a través del sentir, oler, gustar, observar y escuchar los estímulos del contexto). Los niños se apropian de nociones y elaboran conceptos que comparten y complementan con sus pares. Sin embargo, el porcentaje obtenido en la explicación de fenómenos es bajo porque a los niños les cuesta trabajo expresar de manera espontánea sus pensamientos, sentimientos y conocimientos, ya que no saben la manera en que van a ser recibidos por otros. De igual forma, la capacidad para interpretar con claridad y seguir correctamente instrucciones dadas en forma verbal o escrita es baja, puesto que su entorno está lleno de estímulos y les es difícil centrar su atención.

En el nivel de supervisión, el porcentaje más alto se encuentra en la explicación de fenómenos. Esto en razón a que los niños elaboran sus propios conceptos de acuerdo con las experiencias vividas, de manera que pueden expresar sus puntos de vista con respecto a una situación o fenómeno específico con la posibilidad de cambiar o mejorar estos conceptos (de acuerdo con Piaget, citado por Labinowicz, los niños dentro del proceso de asimilación pueden adquirir nueva información y modificar o complementar la ya existente). En segundo lugar, teniendo en cuenta el porcentaje, se encuentra la curiosidad. Los niños necesitan manipular los objetos de su entorno a fin de adquirir conocimientos. Por sus características lo hacen de manera precisa, y además lo disfrutan. Sin embargo, tienen dificultades cuando se les sugiere organizar lo que han aprendido de manera puntual en un registro de observación dirigido, porque dar el paso de tocar los objetos (que son algo concreto), a lo abstracto de un formato, requiere un nivel adecuado de interpretación y atención, el cual, si no se tiene, no se logra un trabajo coherente y descriptivo. Por último, el porcentaje en este nivel desciende en cuanto al trabajo en equipo, puesto que para los niños es muy importante ser tenidos en cuenta a través de sus participaciones y consideran tener siempre la razón, lo cual es un obstáculo para la escucha atenta y respetuosa de sus pares. Aún les es difícil dejar a un lado el egocentrismo y reconocer que en el otro pueden encontrar el complemento a sus experiencias y, por ende, el enriquecimiento intelectual. Por otra parte, aunque los docentes reconocen la importancia de esta estrategia (trabajo en equipo), pocas veces lo permiten; dado que por las características de los niños mencionadas anteriormente, resulta difícil alcanzar de manera satisfactoria los objetivos propuestos durante el desarrollo de actividades mientras se trabaja en equipo.

En el nivel de asesoría, los porcentajes encontrados con respecto a la ejecución y a la supervisión decrecieron. En la categoría de curiosidad, se encuentra que los niños experimentan espontáneamente con los elementos de su entorno; no obstante, cuando la interacción con estos deja de ser libre y pasa a ser dirigida (deben seguir instrucciones claras y precisas), la curiosidad disminuye. Como ya se dijo, les cuesta interpretar dichas instrucciones. Asimismo, a partir de sus experiencias, los niños deben realizar predicciones, las cuales de acuerdo con Piaget, citado por Labinowicz, serían las inferencias que pueden hacer luego de experimentar situaciones en un contexto. Contrario a esto, se evidencia que les cuesta trabajo establecer relaciones entre las situaciones y los fenómenos y, por consiguiente, mantener la coherencia. Justamente por esto se ve afectada la explicación de fenómenos. Adicionalmente, si bien los niños piensan de manera lógica, son fácilmente influenciables, es decir, toman las ideas de otros para expresarlas aun 
cuando no sean apropiadas. En este nivel, el trabajo en equipo se dificulta porque los niños deben darse la oportunidad de interpretar a sus pares, así como de escuchar sus opiniones. La característica del razonamiento inductivo a través de la observación (no sólo de su contexto sino de sus miembros), aunque es propia de los niños de estas edades, no se evidencia, precisamente por las dificultades en la interrelación con los otros (escucha, respeto y tolerancia).

Al nivel de mentalización (nivel de mayor evolución tricerebral), corresponden los porcentajes más bajos obtenidos para las tres actitudes. En la curiosidad, los niños experimentan de manera direccionada, ya que deben interactuar con el entorno con un propósito definido. Aunque los niños están en capacidad de establecer un balance entre sus experiencias nuevas y sus estructuras internas, al hacerlo encuentran obstáculos debido a que se quedan en lo básico, les cuesta trabajo establecer interrelaciones y argumentarlas. Por esta misma razón dentro de la explicación de fenómenos se quedan cortos, no logran expresarse de manera organizada: simplemente emiten ideas con respecto a situaciones o fenómenos de manera suelta y aislada. Del mismo modo, sus respuestas son sencillas, pobres de vocabulario y en consecuencia limitadas en su significado. En el trabajo en equipo, se observa dificultad para organizarse, dado que los niños siempre creen tener la razón. Se dedican a hablar y a discutir sobre la misma idea, en la interacción con sus pares difícilmente llegan a concertar y sus producciones no son enriquecidas con las opiniones y experiencias de sus compañeros.

En cuanto a la curiosidad, los niños manifiestan un interés especial por las ciencias naturales. Evidencian capacidad de asombro ante lo nuevo, por explorar y en la mayoría de casos por manipular objetos del entorno inmediato. Esta actitud se puede apreciar mejor cuando las actividades son realizadas al aire libre o cuando se cambia el espacio habitual, pues el aula limita su espontaneidad ya que implica mayor control del docente y existen unas reglas preestablecidas y constantes (por ejemplo, no levantarse del puesto, no correr, esperar el turno para hablar). En síntesis, el cambio de espacio genera motivación e interés por explorar y conocer.

El trabajo en equipo es el que menos nivel de desarrollo presenta, ya que para que sea viable requiere de la interacción directa con otros y la puesta en escena de valores tales como la escucha, el respeto por el otro y la tolerancia, a fin de generar discusiones sanas que permitan llegar a acuerdos. Pese a que la institución promueve dichos valores, estos se cultivan desde la familia y, en este contexto, se observa que muchas de estas no dan relevancia a la formación en valores. Por esta razón, aún son evidentes en el colegio agresiones verbales a los compañeros y discriminación por género, raza o capacidad cognitiva.

Acerca de la explicación de fenómenos, la mayoría expresa ideas, pensamientos y nociones o conceptos justificados desde su experiencia. Sin embargo, algunas de las explicaciones que dan no son coherentes y no están relacionadas con los conocimientos adquiridos a lo largo de la vida escolar. Estos niños son impulsivos en sus apreciaciones, no se detienen a escuchar lo que se les pregunta y, por consiguiente, dan respuestas inmediatas y sin elaboración.

Respecto a los docentes, es evidente que si bien reconocen la importancia de utilizar diferentes espacios para el desarrollo de las clases, no lo implementan porque temen perder el control del grupo. Como consecuencia, los estudiantes no tienen los espacios que les permitan avanzar a los siguientes niveles de asesoría y mentalización, en los cuales se podría percibir la transformación o generación de nuevos conocimientos mediante los procesos de las estructuras mentales planteadas por Piaget: asimilación, acomodación y adaptación.

Las condiciones del grupo en relación con el número de estudiantes por clase (30 estudiantes) no permiten crear un ambiente apropiado para que cada uno de estos exponga sus ideas. Esto sucede porque los niños, generalmente, manejan tiempos cortos de atención y concentración, de manera que, luego de cuatro o cinco intervenciones de sus compañeros, pierden el interés y se ocupan en otras cosas. Además, no tienen hábitos de escucha y esto genera dificultad para avanzar a los niveles de asesoría y mentalización.

En razón a su corta edad, los niños necesitan orientación y acompañamiento constante durante el desarrollo de las actividades y las experiencias propuestas en el aula y fuera de ella. Si bien suelen dar respuesta a todos los estímulos recibidos o a las respuestas solicitadas a través de su pensar, sentir y actuar, para ello no tienen un orden predeterminado: lo hacen de manera individual y generalmente influenciada unas con otras, es decir, su pensar influye sobre su sentir y su actuar o viceversa.

La autoconducción, autoinformación y autoorganización como elementos de la perspectiva tricerebral pueden observarse de manera individual en los niños, ya que las actividades sugeridas para la caracterización de las actitudes científicas (curiosidad, 
trabajo en equipo y explicación de fenómenos), causaron un impacto progresivo acorde con la evolución tricerebral. Es preciso agregar cómo estas actividades rompieron con la cotidianidad de los niños, y dejaron atrás algunos esquemas trabajados con ellos durante las clases de ciencias naturales.

\section{Conclusiones}

La teoría tricerebral de Gregori aporta al conocimiento profundo de los procesos de enseñanza-aprendizaje. Permite ver a los niños y niñas como seres integrales, con la posibilidad de potenciar todas sus habilidades y competencias desde la escuela. La aplicación del registro de observación y el revelador del cociente tríadico, en conjunto, permiten registrar de manera objetiva y puntual el nivel de los procesos de cada uno de los estudiantes $y$, a su vez, del grupo, lo cual se constituye en una herramienta valiosa para los docentes al momento de generar estrategias metodológicas, enriqueciendo cada vez más la práctica pedagógica.

El fortalecimiento de las actitudes científicas a través de la enseñanza de las ciencias naturales permite estructurar el pensamiento no sólo científico, sino crítico y reflexivo, con el fin de mejorar la resolución de problemas de la vida cotidiana e incentivar la sana convivencia dentro y fuera de la escuela.

Estimular de manera constante las actitudes científicas en los niños les permite desenvolverse de manera asertiva en un mundo cambiante que, día tras día, presenta mayor nivel de exigencia para transformarlo. Asimismo, aporta a la formación de un semillero orientado a la interpretación crítica de realidades que conllevan la transformación social, cultural y ambiental.

La posibilidad de transformar y mejorar el mundo está en manos de los niños y las niñas de hoy. Ellos ven en la escuela un lugar de juego, aprendizaje y conocimiento, donde establecen una relación directa con su maestro, el cual debe reconocer en sus estudiantes las actitudes científicas y plantear estrategias para fortalecerlas. Con este fin se hace necesario construir una relación horizontal de los pares con sus estudiantes, dado que a través de las emociones el aprendizaje se hace más significativo y duradero.

Es indispensable diseñar programas curriculares que se centren en el desarrollo de actitudes científicas y no en contenidos, puesto que estos tienden a olvidarse si no se aprenden de una manera significativa.
La curiosidad, el trabajo en equipo y la explicación de fenómenos permiten un acercamiento al conocimiento desde una perspectiva diferente, duradera y estimulante. Si bien estos nacen en el seno de las ciencias naturales, son el fundamento y la base del conocimiento formal, el cual inicia en la infancia, pero se fortalece a lo largo de los años de escolaridad y se hace visible en todos los campos del conocimiento.

Es fundamental cambiar la tradición de la enseñanza, que aún en la actualidad se rige por el paradigma formal (cerebro derecho e izquierdo). Es decir, los docentes deben transformar las prácticas pedagógicas a través de procesos de reflexión y acción, lo cual les permita innovar en la escuela - en este caso, aplicar la teoría tricerebral de De Gregori como alternativa de cambio-, en beneficio de la calidad de la educación.

\section{Referencias}

Daza, Silvio. La enseñanza de las ciencias naturales en las primeras edades. Barrancabermeja: Greci Inbuya, 2011. Impreso.

De Gregori, Waldemar. Construcción familiar-escolar de los tres cerebros. Bogotá: Editorial Kimpres Ltda., 2002. Impreso.

Giordán, André. Enseñanza de las ciencias naturales: la curiosidad. México: Secretaría de Educación de México, 2001. Web.

Harlen, Wynne. Enseñanza y aprendizaje de las ciencias. Madrid: Ediciones Morata, 1994.

Hernández Sampieri, Roberto, Carlos Fernández Collado y María Baptista Lucio. Metodología de la Investigación. México: McGraw-Hill, 2010. Web.

Instituto Colombiano para el Fomento de la Educación Superior (ICFES). Fundamentación conceptual área de ciencias naturales. Bogotá: Grupo de Procesos Editoriales ICFES, 2007. Impreso.

Johnson, David W, Roger Johnson y Edythe Holubec. El aprendizaje cooperativo en el aula. Argentina: Talleres Gráficos D’Aversa, 1999.

Labinowicz, Ed. Introducción a Piaget. Pensamiento. Aprendizaje. Enseñanza. México: Editorial Adison Wesley Longman, 1998. Web.

Piaget, Jean. Seis estudios de psicología. Trad. Jordi Márfa. Editorial Labor, 1991. Web.

Velandia Mora, Manuel Antonio. Trabajo en equipo. España, 2013. Web. 